\title{
Development and Application of Mass
} Spectroscopy Assays for $\mathrm{N}^{\varepsilon}$-(1-Carboxymethyl)L-Lysine and Pentosidine in Renal Failure and Diabetes

\author{
Katherine L. O'Grady, Sundeep Khosla*, Joshua N. Farr, Olga P. Bondar, Elizabeth J. Atkinson, \\ Sara J. Achenbach, Brittany A. Eckhardt, Brianne S. Thicke, Amanda J. Tweed, \\ Tammie L. Volkman, Matthew T. Drake, Jolaine M. Hines, and Ravinder J. Singh*
}

Background: Advanced glycation end products (AGEs) are formed via the nonenzymatic glycation of sugars with amino acids. Two AGEs, $\mathrm{N}^{\varepsilon}$-(1-carboxymethyl)-L-Lysine (CML) and pentosidine, have been observed to be elevated in subjects suffering from a multitude of chronic disease states, and accumulation of these compounds may be related to the pathophysiology of disease progression and aging.

Methods: We describe here the development and validation of a specific and reproducible LC-MS/MS method to quantify CML and pentosidine in human serum with lower limits of quantitation of $75 \mathrm{ng} / \mathrm{mL}$ and $5 \mathrm{ng} / \mathrm{mL}$, respectively. The analyte calibration curve exhibited excellent linearity at a range of 0-10 900 ng/mL for CML and 0$800 \mathrm{ng} / \mathrm{mL}$ for pentosidine. High-low linearity of 5 serum pairs was assessed, with a mean recovery of $103 \%$ (range 94-116\%) for CML, and 104\% (range 97-116\%) for pentosidine.

Results: Serum concentrations of CML and pentosidine were quantified in 30 control and 30 subjects with chronic renal insufficiency. A significant increase in both analytes was observed in renal failure compared to control subjects (2.1-fold and 8.4-fold, respectively; $P<0.001$ for both). In a separate cohort of 49 control versus 95 subjects with type 2 diabetes mellitus (T2DM), serum CML but not serum pentosidine, was significantly elevated in the T2DM patients, and CML was also correlated with glycemic control, as assessed by hemoglobin A1c $(r=0.34$, $P<0.001$ ).

Conclusions: These mass spectroscopy-based assays for serum CML and pentosidine should be useful in accurately evaluating circulating levels of these key AGEs in various disease states.

Mayo Clinic College of Medicine, Rochester, MN.

*Address correspondence to: S.K. at College of Medicine, Mayo Clinic, 200 First Street SW, Rochester, MN 55905. Fax: 507-293-3853; e-mail khosla. sundeep@mayo.edu. R.J.S. at College of Medicine, Mayo Clinic, 200 First Street SW, Rochester, MN 55905. Fax 507-293-3853; e-mail singh.ravinder@mayo.edu

Received August 27, 2019; accepted November 19, 2019.

DOI: 10.1093/jalm/jfaa023

(c) American Association for Clinical Chemistry 2020. All rights reserved.

For permissions, please email: journals.permissions@oup.com 


\section{IMPACT STATEMENT}

We describe the development of a specific and reproducible mass spectroscopy-based assay for serum advanced glycation endproducts (CML and pentosidine). A significant increase in circulating levels of both analytes was observed in renal failure compared to control subjects; serum CML, but not pentosidine, was elevated in patients with type 2 diabetes mellitus. Given the growing research and clinical interest in the role of AGEs in the pathogenesis of a number of diseases, including the complications of diabetes mellitus and renal failure, these novel assays should be an important tool to advance research and clinical practice in this area.

\section{INTRODUCTION}

Advanced glycation endproducts (AGEs) accumulate in various tissues with aging and may contribute to the pathology of diabetes mellitus, atherosclerosis, and induction of oxidative stress and inflammation $(1,2)$. AGEs are a heterogeneous group of compounds $(3,4)$ formed via a complex pathway known as the Maillard Reaction (5). One condition known to stimulate the production of these compounds is prolonged hyperglycemia, whereby nonenzymatic glycation of reducing sugars with amino acid groups, lipids, or nucleic acids generate AGEs (6). In addition to diabetes, AGEs have also been shown to accumulate in patients with renal failure $(7,8)$. AGEs are thought to have a cascading effect that further increases oxidative stress via free radical damage, which subsequently promotes additional formation of AGEs as well as chronic inflammation.

Although AGEs accumulate in tissues, circulating levels of AGEs may also be useful in monitoring potential tissue complications, exemplified best by the measurement of glycated hemoglobin $(\mathrm{HbA1c})$ and its known relationship to diabetes complications (9). However, in addition to HbA1c, circulating levels of pentosidine and CML have also been measured in patients with diabetes, most commonly using enzyme-linked immunosorbent assays (ELISAS). The use of ELISAS for AGEs has been associated with several problems, however, including: i) the affinity of antibodies can vary for different protein residues; ii) characterization of antibody specificity may be lacking; and iii) differences may exist between the antigens present in biological samples and the highly modified standard antigens used for antibody production (10).

We sought to develop a quantitative method for two AGEs: CML, the most abundant AGE, and pentosidine, without the use of antibodies. In this report we describe the development and clinical validation of a novel assay that is highly specific, sensitive, and reproducible using liquid chromatography combined with tandem mass spectroscopy (LC-MS/MS) for the detection of circulating levels of CML and pentosidine in human serum.

\section{METHODS}

\section{Study Subjects}

The protocol was approved by the Mayo Clinic Institutional Review Board.

Controls versus renal insufficiency/failure patients. Sixty serum samples were collected in control and renal insufficiency/failure groups, each containing 30 subjects (15 male/15 female) 
and renal insufficiency/failure was defined as serum creatinine concentration above $1.2 \mathrm{mg} / \mathrm{dL}$ (the cutoff reference level).

\section{Control versus type 2 diabetes (T2DM)} patients. We recruited postmenopausal women and men $\geq 50$ years old. T2DM subjects were defined as having a HbA1c level $\geq 6.5 \%$, and we studied subjects with T2DM for at least 5 years. Exclusion criteria included: a) significant abnormality in any of the screening laboratory studies [complete blood count, serum calcium $(>10.5 \mathrm{mg} / \mathrm{dL})$, eGFR based on creatinine $\left(<30 \mathrm{~mL} / \mathrm{min} / 1.73 \mathrm{~m}^{2}\right), \mathrm{TSH}(<0.1 \mathrm{mIU} / \mathrm{L})$, and $25-$ hydroxyvitamin $\mathrm{D}(<15 \mathrm{ng} / \mathrm{mL})]$; b) presence of clinically noted chronic liver disease, unstable cardiovascular disease, malignancy, chronic gastrointestinal disease, autoimmune rheumatologic conditions, hypo- or hyperparathyroidism, Cushing's syndrome, severe chronic obstructive pulmonary disease, alcoholism, or type 1 diabetes; c) undergoing treatment with any of the following drugs: corticosteroids ( $>3$ months at any time or $>10$ days within the previous year); treatment within the past year with any of the following: anticonvulsants, pharmacological doses of thyroid hormone, adrenal or anabolic steroids, aromatase inhibitors, calcitonin, estrogen, selective estrogen receptor modulators, parathyroid hormone, denosumab, or thiazolidinediones. We also excluded subjects who have been exposed to a bisphosphonate within the past 5 years.

Materials and methods. $\mathrm{N}^{\varepsilon}-(1$-carboxymethyl)-Llysine $\quad(\mathrm{CML}), \quad \mathrm{N}^{\varepsilon}$-(1-carboxymethyl)-L-lysine-d4 (CML-d4), and pentosidine-d3 trifluoroacetic acid salt were purchased from Toronto Research Chemicals. Pentosidine was obtained from Cayman Chemicals. Hydrochloric acid (36.538.0\%), perfluoropentanoic acid (97\%), and ammonium hydroxide (28-30\%) were purchased from Sigma Aldrich; acetonitrile, methanol, and acetone were obtained from Fisher Scientific.
Stock solutions of both $\mathrm{CML}$ and pentosidine were prepared, separately, in water at $1 \mathrm{mg} / \mathrm{mL}$ and $100 \mu \mathrm{g} / \mathrm{mL}$ concentrations. Calibration curves were constructed from a dilution of an intermediate stock containing $15 \mu \mathrm{g} / \mathrm{mL} \mathrm{CML}$ and $1 \mu \mathrm{g} / \mathrm{mL}$ pentosidine. This intermediate was diluted in water to prepare standards with the following CML/ pentosidine concentration ratios $(\mathrm{ng} / \mathrm{mL})$ : 0/0, 75/ 5, 150/10, 300/20, 750/50, 1500/100, 3000/200, 5250/350, 7500/500, and 10900/800.

Stock solutions of deuterated internal standards, CML-d4 and pentosidine-d3, were prepared, separately, in concentrations of $1 \mathrm{mg} / \mathrm{mL}$. Working solutions were made at $10 \mu \mathrm{g} / \mathrm{mL}$ for CML-d4 and $1 \mu \mathrm{g} / \mathrm{mL}$ for pentosidine-d3.

Quality controls of low, medium, and high concentrations were prepared by diluting pooled human serum 1:2 with nanopure water (low), left neat (medium), or spiked with $+1250 \mathrm{ng} / \mathrm{mL} \mathrm{CML}$ and $+500 \mathrm{ng} / \mathrm{mL}$ pentosidine (high). All three quality controls were run at the beginning and end of each sample batch.

Sample preparation. Standards, controls, and samples were stored frozen prior to use. For hydrolysis, $50 \mu \mathrm{L}$ of control or sample was pipetted into $13 \times 100 \mathrm{~mm}$ borosilicate glass culture tubes with $475 \mu \mathrm{L}$ of $6 \mathrm{M} \mathrm{HCl}$ prepared in water, $25 \mu \mathrm{L}$ of $10 \mu \mathrm{g} / \mathrm{mL} \quad \mathrm{CML}-\mathrm{d} 4$, and $25 \mu \mathrm{L}$ of $1 \mu \mathrm{g} / \mathrm{mL}$ pentosidine-d3. Samples were capped with Teflon lined screw caps, vortexed for $1 \mathrm{~min}$ at $\sim 1500 \mathrm{rpm}$, and hydrolyzed for $20 \mathrm{~h}$ at $110^{\circ} \mathrm{C}$ in a protected heat block. After hydrolysis, samples were cooled to room temperature and centrifuged at $3000 \mathrm{rpm}$ for $10 \mathrm{~min}$ to remove particulates. To extract analytes, $0.6 \mathrm{~mL}$ volume of activated AG50W-X8 resin (Bio-Rad Laboratories) was added to filter tubes in a Cerex-48 SPE ware holder and then washed five times with $1 \mathrm{~mL}$ nanopure water. Hydrolysates were transferred onto the resin using disposable transfer pipets. Each filter tube was washed five times with $2 \mathrm{~mL}$ nanopure water, then 
CML and pentosidine were eluted into $13 \times 100$ $\mathrm{mm}$ glass tubes with 3 consecutive additions of $1 \mathrm{~mL} 4 \mathrm{M} \mathrm{NH}_{4} \mathrm{OH}$. Extracts were dried down under nitrogen at $50^{\circ} \mathrm{C}$ and $15-20 \mathrm{psi}$ for $2-3 \mathrm{~h}$ in a Zymark Turbo Vap water bath. Once dry, extracts were reconstituted with 90:10 water: acetonitrile with $5 \mathrm{mM}$ perfluoropentanoic acid (NFPA), vortexed for 2 min, filtered into 96-well collection plates, and stored refrigerated until analysis.

LC-MS/MS. A triple-quadrupole mass spectrometer, Sciex API 5000 (Applied Biosystems) utilizing an electrospray ionization source (ESI) in positive ion mode was used in combination with a Cohesive TLX4 liquid chromatography system. Ion-pairing chromatography was performed on a Phenomenex Synergi $4 \mu$, Max RP 80 A, $2.0 \times 150 \mathrm{~mm}$ analytical column with a $C_{12}, 4 \times 2 \mathrm{~mm}$ guard cartridge (Phenomenex). The injection volume was $75 \mu \mathrm{L}$. Mobile phases were prepared using NFPA as the ion-pairing agent. Mobile Phase A contained 5 mM NFPA in 90:10 water: acetonitrile, while Mobile Phase $B$ consisted of $5 \mathrm{mM}$ NFPA in 10:90 water: acetonitrile. Mobile phases were degassed for 5 min prior to use. The liquid chromatography method is detailed in Supplemental Table 1. The total method length was $19.25 \mathrm{~min}$, with analyte retention times of $\sim 7.3 \mathrm{~min}$ for $C M L$, and $\sim 8.8 \mathrm{~min}$ for pentosidine. Flow was diverted from the mass spectrometer for the first 5 min of chromatography to prevent contamination.

Ionization was performed in positive mode with multiple reaction monitoring (MRM) transitions. Mass transitions and compound parameters used for CML and pentosidine and the associated internal standards are included in Supplemental Table 2 while source and gas settings are detailed in supplemental Table 3.

\section{Total Advanced Glycation Endproducts (AGEs)}

Prior to addition of samples, the background of an empty, black microtiter plate was acquired and wells with greater than $100 \mathrm{AU}$ (arbitrary units) were excluded for use in analysis. For Total AGEs analysis using fluorescence detection, samples were diluted 5 times with PBS ( $\mathrm{pH} 7.4)$ and $300 \mu \mathrm{L}$ of the diluted sample was added to the plate. The samples were read at excitation wavelength of $370 \mathrm{~nm}$ and emission wavelength of $440 \mathrm{~nm}$ using a Spectra Max Gemini XS (Molecular Devices). The fluorescence was normalized to $C M L$, used as a surrogate for total AGES.

Biochemical assays and estimated glomerular filtration rate (GFR). $\mathrm{HbA} 1 \mathrm{c}$ and creatinine were measured in the Mayo Clinical Laboratory by ionexchange high-performance liquid chromatography and an enzymatic colorimetric assay, respectively. The CKD-Epi equation was used to estimate GFR based on age, sex, race, and serum creatinine levels (11).

Skin AGEs measurements. Skin AGEs were measured using the AGE Reader ${ }^{\circledR}$ (Diagnoptics), which measures an index of tissue accumulation of AGEs by means of skin autofluorescence (12). The AGE reader measures skin autofluorescence with an excitation light source with wavelength between 300 and $420 \mathrm{~nm}$. Previous studies have shown an error rate of $\sim 5 \%$ when repeated skin autofluorescence measurements are made in control and T2DM patients (12).

Statistical analyses. Clinical characteristics of the various cohorts were summarized using medians and interquartile ranges. Chi-square and KruskalWallis tests were used to assess group differences for categorical and continuous variables. Linear regression models were used to test for group differences in CML, pentosidine, and total AGEs values adjusting for eGFR values. Spearman correlations were assessed to compare the various lab measurements. 


\section{RESULTS}

\section{Quantification of CML and pentosidine}

Standards spiked with CML-d4 and pentosidine-d3 internal standards were analyzed via LC-MS/MS. Using linear regression analysis, calibration curves were found to be extremely reproducible over 6 days (Fig. 1) with results for $\mathrm{CML}$ and pentosidine presenting slopes of 1.0014 and 1.0061, and $r^{2}$ values of 0.997 and 0.9999 , respectively.

Chromatographic separation of CML and pentosidine was achieved using an ion-pairing reagent combined with reversed-phase chromatography. This facilitated optimization of peak shapes for both analytes and their internal standards (Fig. 2).

To verify precision, both intra- and inter-assay imprecision was performed. Intra-assay imprecision was determined by running 3 levels of quality control 20 times within the same assay. Interassay imprecision was determined by running 3 levels of quality control material over the course of 8 days. Values of the controls spanned the analytical measurable range and results are listed in Supplemental Table 4.

Spiked recovery and admixture experiments were performed to ensure accuracy. CML and pentosidine were spiked into human serum in varied amounts. CML was spiked at 300, 750, and $1500 \mathrm{ng} / \mathrm{mL}$ concentrations with mean recovery ranging from 96-102\%. Pentosidine was spiked at 20,50 , and $100 \mathrm{ng} / \mathrm{mL}$ concentrations with mean recovery ranging from 92-104\%. High: low admixture studies were performed in ratios of 90:10, 75:25, 50:50, 25:75, and 10:90 for 5 pairs of serum samples. Recoveries ranged from 94-116\% (mean 104\%) for CML (645-7080 ng/mL)and 97-116\% (mean 104\%) for pentosidine $(5.4-389 \mathrm{ng} / \mathrm{mL}$ ). Dilutional linearity experiments were performed on 6 different samples diluted with water $\times 2, \times 5$, and $\times 10$. The series showed excellent linearity with measured/expected values ranging from 81-

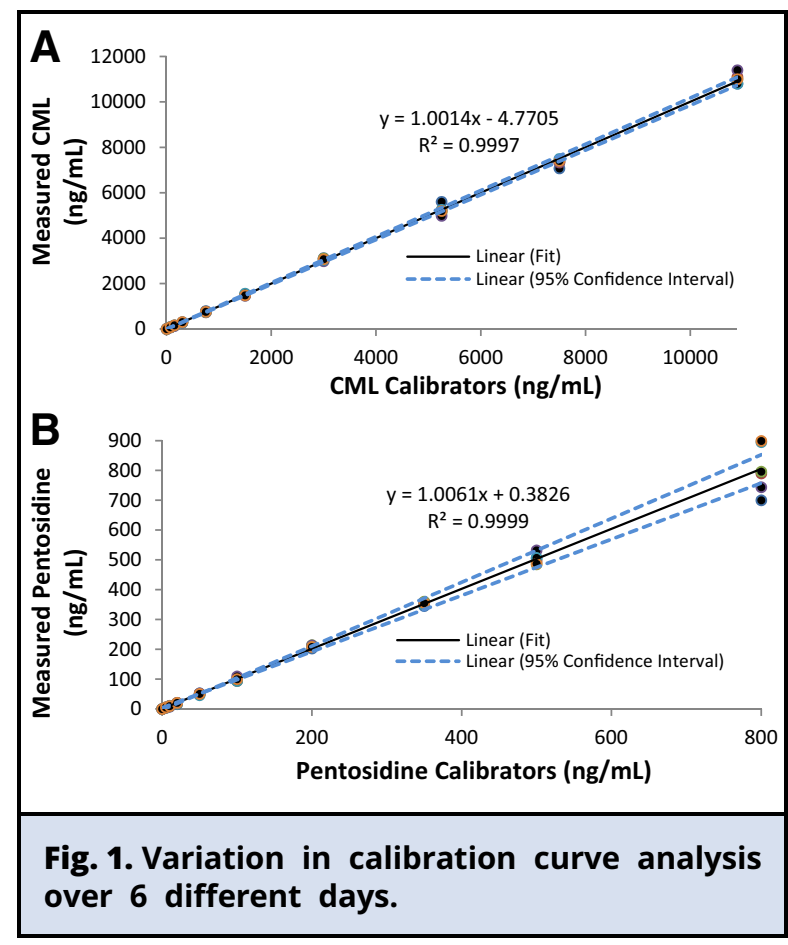

110\% (mean 93\% for values 230-16 $800 \mathrm{ng} / \mathrm{mL}$ ) for CML and $87-114 \%$ (mean 99\% for values 5.8 $534 \mathrm{ng} / \mathrm{mL}$ ) for pentosidine.

\section{Control versus renal failure patients}

We next evaluated $C M L$, pentosidine, and total AGEs in a cohort of control versus renal failure patients. Supplemental Table 5 shows the clinical characteristics of the control and renal failure patients. Fig. 3, A-C shows the values for $C M L$, pentosidine, and total AGEs in the control versus renal failure patients. As is evident, all 3 AGEs parameters were markedly higher in the renal failure as compared to the controls. Table 1 shows the correlations between $C M L$, pentosidine, and serum AGEs in the renal failure and control subjects. CML and pentosidine were strongly correlated in both groups. Total serum AGEs were correlated with CML and pentosidine in the renal failure but not the control group. 

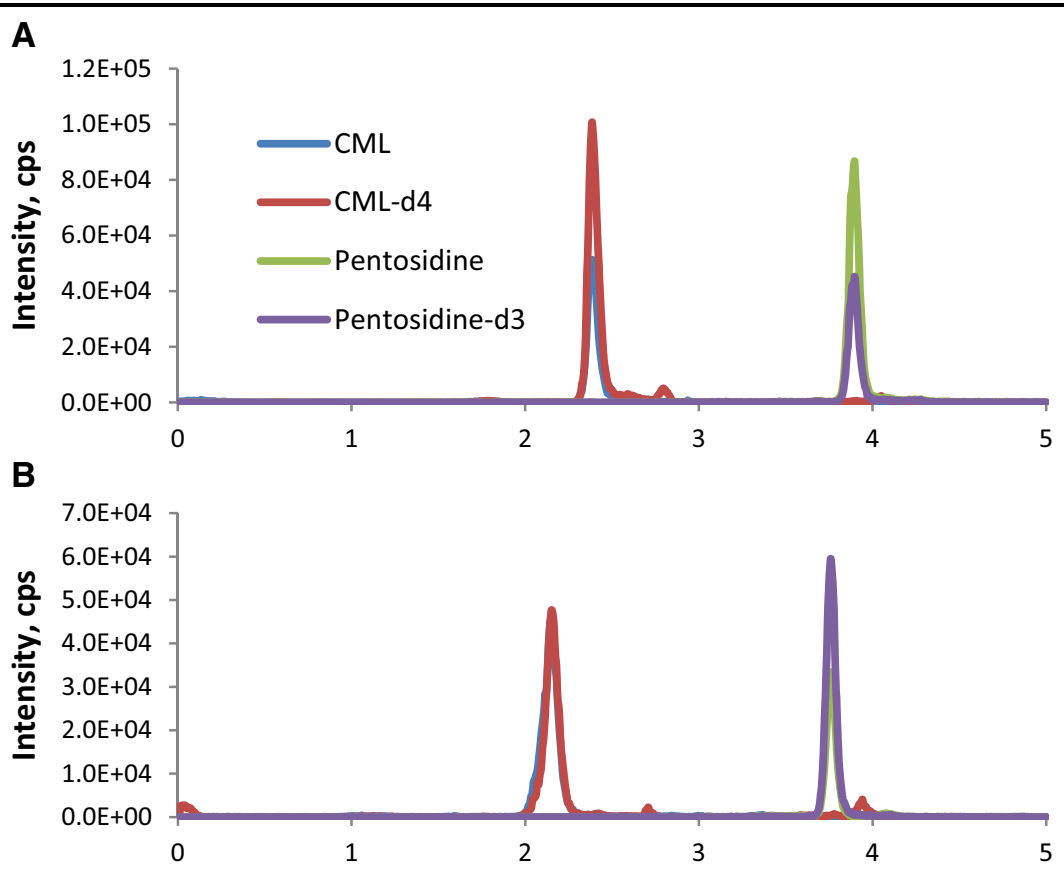

Fig. 2. Example of $\mathrm{CML}$ and pentosidine chromatographic peak shape. a) Spiked human serum sample, $\mathrm{CML} 5300 \mathrm{ng} / \mathrm{mL}$ and pentosidine $699 \mathrm{ng} / \mathrm{mL}$; b) Neat serum from renal failure subject, $\mathrm{CML}=7970 \mathrm{ng} /$ $\mathrm{mL}$ and pentosidine $398 \mathrm{ng} / \mathrm{mL}$.

\section{Control versus T2DM patients}

We also evaluated $C M L$, pentosidine, and total AGEs in a second cohort consisting of control versus T2DM patients. Supplemental Table 6 shows the clinical characteristics of the control and T2DM patients used in these analyses. The median duration of T2DM was 14years (range, 530 years). The median $\mathrm{HbA} 1 \mathrm{c}$ over the preceding 5 years in the T2DM patients was 7.4\%, with a fairly wide range (6.5-11.4\%). Skin AGEs were significantly higher in the T2DM patients. Serum creatinine and eGFR did not differ between the control and T2DM subjects.

Fig. 4 shows the $C M L$, pentosidine, and total AGEs values in the control versus T2DM patients. CML was significantly higher in the T2DM as compared to the control subjects, whereas serum total AGEs or pentosidine were not different between the 2 groups. Since a previous analysis had suggested that CML was elevated only in T2DM patients with renal insufficiency (13), we repeated the comparisons in Fig. 4 following adjustment for eGFR, with very similar results (data not shown). We further examined the group of T2DM patients with the worst glycemic control (median 5 year $\mathrm{HbA} 1 \mathrm{C}>8 \% ; N=30$ ), with very similar results: CML (median [IQR]) was higher in the T2DM (2110 [1700-2370] ng/mL) versus the control subjects [1540 (1350-1720) ng/mL, $P<0.001]$, whereas pentosidine (T2DM, 17.1 [14.1-19.2] versus control, 15.6 [12.9-20.9] ng/mL, $P=0.68)$ and serum AGEs (T2DM, 0.93 [0.86-1.01] versus control, 0.96 [0.89-1.05] $\mu \mathrm{M}, P=0.42$ ) did not differ between groups.

Table 2 shows the correlations between serum $C M L$, pentosidine, and $A G E s$, as well as the other 

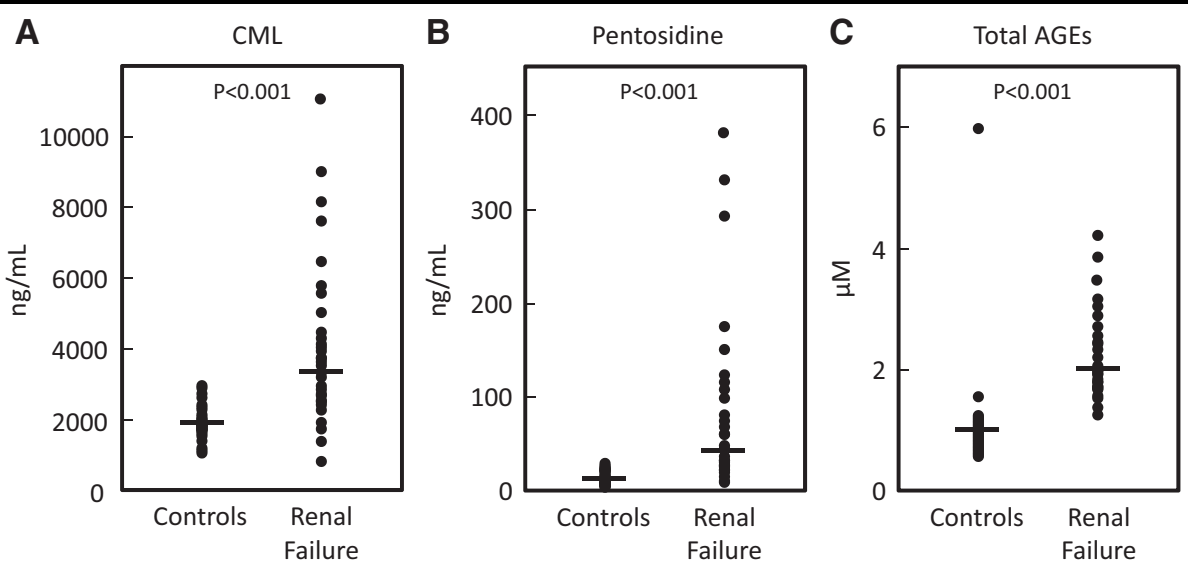

Fig. 3. Serum a) CML, b) pentosidine, and c) total AGEs concentrations in control versus renal failure patients. Bars indicate median values.

Table 1. Spearman correlation coefficients adjusted for age and sex between CML, pentosidine, and serum AGEs in the renal failure and control subjects.

\begin{tabular}{|c|c|c|c|c|}
\hline & \multicolumn{2}{|c|}{ Renal failure } & \multicolumn{2}{|c|}{ Control } \\
\hline & CML & Pentosidine & CML & Pentosidine \\
\hline CML & 1.0 & $0.87^{* \star \star}$ & 1.0 & $0.54^{* *}$ \\
\hline Serum AGEs & $0.69^{\star \star \star}$ & $0.48^{* *}$ & 0.13 & 0.20 \\
\hline
\end{tabular}

variables (median HbA1c over 5 years, and skin AGES). CML was correlated with pentosidine and $\mathrm{HbA1c}$ in both the control and T2DM subjects. CML did not correlate with serum AGEs or skin AGEs in either group. Pentosidine was correlated with CML in both groups and with serum AGEs in the T2DM subjects. Serum AGEs correlated with pentosidine and skin AGEs in the T2DM but not the control subjects.

\section{Comparison of changes relative to controls in the disease cohorts}

Supplemental Table 7 compares the fold increases in serum CML, pentosidine, and total
AGEs in the renal failure and T2DM cohorts. As shown, although the 1.2-fold increase in serum CML in the T2DM subjects was statistically significant (Fig. 4), it was much smaller than the fold increase in CML (2.1) in the renal failure patients, who also had a marked increase (8.4-fold) in serum pentosidine; the latter did not change in the T2DM subjects. Total AGEs were also increased 1.9-fold in the renal failure patients, but did not change in the T2DM patients.

\section{DISCUSSION}

Utilizing LC-MS/MS, we developed a novel, reproducible assay to detect and quantitate two select AGEs, CML and pentosidine in serum. Although previous studies have described mass spectroscopy-based assays for CML and/or pentosidine (14-16) and discussed issues related to mass spectrometric detection of these compounds in clinical samples $(17,18)$, our study provides extensive validation of our assay that we can now offer to the scientific community as an orderable test in a College of American Pathologists (CAP)- and Clinical Laboratory Improvement Amendments (CLIA)-certified laboratory. In 


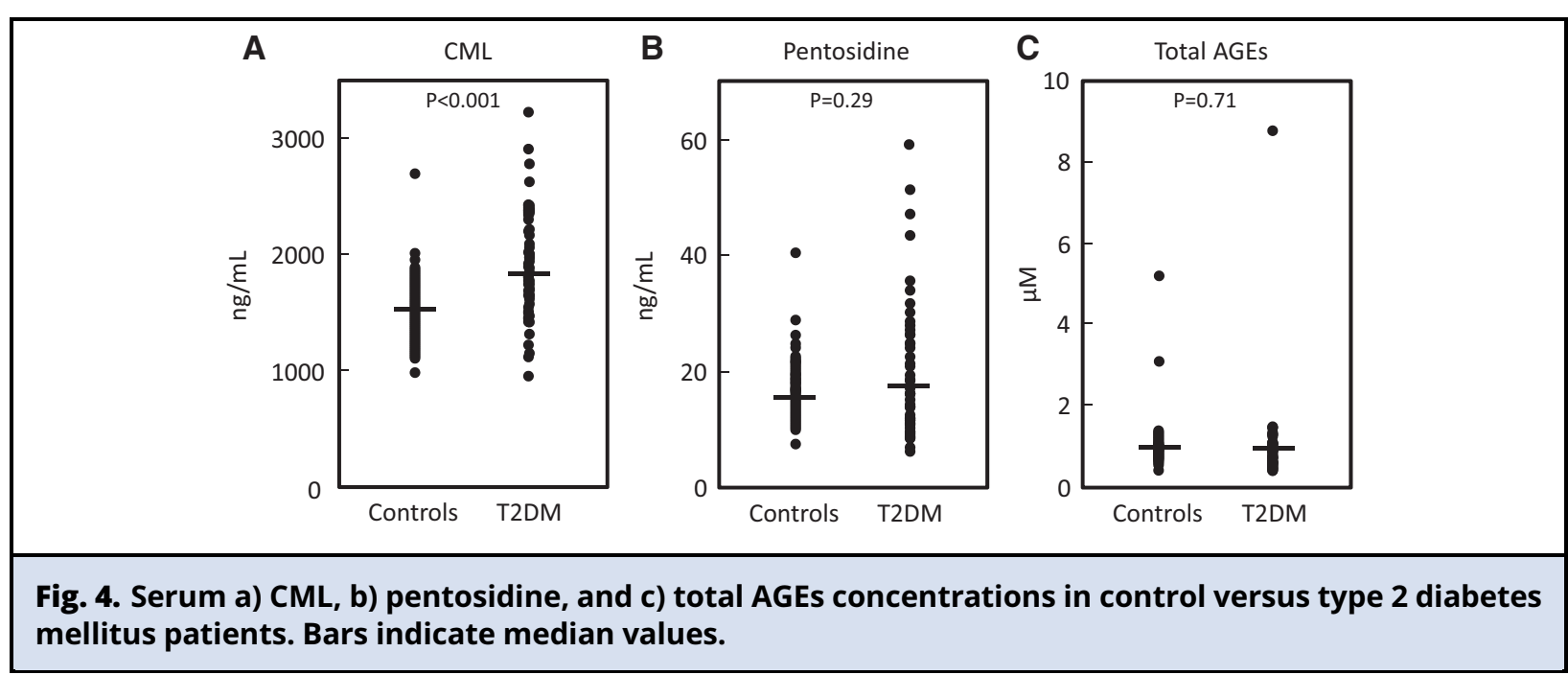

Table 2. Spearman correlation coefficients adjusted for age, sex, and BMI in the control and T2DM patients.

\begin{tabular}{|c|c|c|c|c|c|}
\hline Control & CML & Pentosidine & Serum AGEs & HbA1c & Skin AGEs \\
\hline CML & 1.0 & $0.70^{* \star \star}$ & 0.05 & $0.30^{*}$ & 0.11 \\
\hline Pentosidine & $0.70^{* * *}$ & 1.0 & 0.04 & 0.13 & 0.12 \\
\hline Serum AGEs & 0.05 & 0.04 & 1.0 & 0.01 & -0.19 \\
\hline \multicolumn{6}{|l|}{ T2DM } \\
\hline CML & 1.0 & $0.47^{* * *}$ & 0.19 & $0.34^{* \star *}$ & -0.11 \\
\hline Pentosidine & $0.47^{\star \star \star}$ & 1.0 & $0.41^{* \star *}$ & 0.02 & 0.16 \\
\hline Serum AGEs & 0.19 & $0.41^{* \star *}$ & 1.0 & -0.10 & $0.37^{* * *}$ \\
\hline
\end{tabular}

addition, our simultaneous evaluation of $\mathrm{CML}$, pentosidine, and total AGEs in control versus renal failure and T2DM patients provides additional validation to our assay and shows the relationships between these AGEs and other parameters, including $\mathrm{HbA} 1 \mathrm{C}$ and skin AGEs, in the diabetic cohort.

Using LC-MS/MS for the quantification of AGES is more sensitive and specific than fluorescence or ELISAs, due to instrumentation specificity and capacity to avoid quantitating miscellaneous interference signals. Preliminary fluorescence studies on renal failure subject hydrolysate did not correlate linearly with LC-MS/MS values, and we postulate that untargeted, fluorescing compounds in the matrix are likely to blame. Hydrolysis, extraction, and chromatography were optimized for both analytes to successfully eliminate interference peaks and reduce ion suppression. Inter-assay ( $n=15)$ and intra-assay $(n=20)$ imprecision are acceptable for both analytes. Spiked recovery, dilutional linearity, and admixture studies performed all met our standardized criteria $(<20 \%$ CV).

In serum, pentosidine is primarily proteinbound; thus, only extremely low concentrations of 
free pentosidine are present prior to acid hydrolysis (19). In order to quantitate CML and pentosidine accurately using an LC-MS/MS method, protein conjugated analytes must be cleaved off before detectable levels are achieved; therefore, hydrolysis is a crucial step in sample preparation prior to LC-MS/MS analysis. Hydrochloric acid hydrolysis is one of the most commonly reported methods used to free CML and pentosidine, due to the acid stability of these compounds (14). Kinetics studies that determined analyte concentrations present in serum matrix at a hydrolysis time spanning from zero to $24 \mathrm{~h}$ were undertaken to refine our hydrolysis method, and it was concluded that a $20 \mathrm{~h}$ acid hydrolysis was optimal for both analytes, as both CML and pentosidine plateaued in their response (Supplemental Fig. 1). Additional experiments were conducted to determine the most efficient hydrolysis procedure with regard to the volumetric ratio of serum to acid, and differences between varying molarities of hydrochloric acid. The final method is a representation of the conclusions reached from the above preliminary experiments.

With regard to assay performance, it is important to note that each batch requires substantial preparation prior to injection (30-32 h), which is tedious and may interfere with standard laboratory workflow. A significant amount of time is required to prepare daily/weekly reagents and to maintain proper functioning of equipment. Diligent efforts to replace guard cartridges and monitor changes in chromatography are needed to ensure proper sample processing. Further research must be conducted to determine whether a build-up from particulates in the sample matrix, or degradation to columns and cartridges due to the acidity of mobile phases are behind the previously stated nuances. In the case this assay is used in a clinical setting, subsequent efforts to reduce method duration may prove to be beneficial.

AGEs have been found to be significantly elevated in the serum, tissue, and bones of subjects suffering from a variety of chronic disease states. Pentosidine and CML have been well characterized as biomarkers for the formation and accumulation of AGEs (20), and are known to play an important role in diabetes and vascular complications $(21,22)$. In our study, serum CML was significantly elevated in the diabetic subjects, and was found to correlate with glycemic control measured by $\mathrm{HbA1c}$. However, no significant difference in pentosidine concentration was observed between the T2DM cohort and controls. In contrast with our study, Yoshida et al. found that serum pentosidine concentrations were significantly higher in patients with diabetes than in age-matched control subjects (23). These differing findings may reflect the fact that Yoshida et al. (23) used an immunoassay, rather than mass spectrometry, to measure serum pentosidine and thus their assay may not have been entirely specific for pentosidine. Since both serum pentosidine (24) and CML (13) may be altered by renal function, we also performed analyses adjusting for eGFR. After adjustment for eGFR, there remained no significant difference in pentosidine between diabetic and healthy subjects. Conversely, CML concentrations were significantly higher in the diabetic cohort relative to nondiabetic control subjects, both with and without adjustment for eGFR, and in both the T2DM and control cohorts, CML concentrations correlated with $\mathrm{HbA} 1 \mathrm{c}$.

Analyte concentrations were also determined in a separate cohort of 30 normal subjects and 30 subjects with renal failure. A significant increase in both analytes was observed in the renal failure cohort compared to controls. Prior studies have documented a dramatic accumulation of pentosidine in the plasma of subjects with end-stage renal disease (25), likely due, at least in part, to decreased renal elimination (26). A study utilizing stable isotope mass spectrometry found both CML and carboxyethyl-lysine (CEL) to be significantly increased in dialysis patients compared to controls (14). The accumulation of CML and 
pentosidine in chronic kidney disease is likely due to a lack of excretion from the kidneys and increased production of AGEs from chronic inflammation and oxidative stress. Mounting evidence indicates that under chronic hyperglycemic or renal insufficiency conditions, the accumulation of AGEs, which are consequences of nonenzymatic reactions between sugar and free amino acid groups of proteins, lipids, and nucleic acids, promote the generation of reactive oxygen species (ROS), leading to oxidative stress and additional AGE production (27). One possible consequence of elevated synthesis of AGEs may paradoxically be irreversible damage to the kidney tissues over time via overproduction of ROS (27).

In this study we observed that skin AGEs measured by autofluorescence were markedly higher in the renal failure cohort compared to controls. Skin AGEs were also found to be significantly higher in the T2DM cohort compared to controls (28). In our study, serum creatinine and eGFR did not differ between the control and T2DM group, which demonstrates that T2DM serves as a separate risk factor for AGE accumulation in the absence of impaired renal function. Prior studies have shown that patients with diabetes currently on hemodialysis have even higher skin autofluorescence values than diabetes patients without impaired renal function, which supports the conclusion that both T2DM and end stage renal disease are independent risk factors for AGE accumulation (28).

In comparing the fold increases in serum CML, pentosidine, and total AGEs in the renal failure versus T2DM patients, we found that the magnitude of the increases were much greater and included all 3 analytes in the renal failure patients, whereas only serum CML was significantly elevated in the T2DM patients. Whether this is due to a greater production or decreased clearance (or both) of AGEs in renal failure versus T2DM requires further evaluation.

In summary, we have developed and clinically validated a specific and reproducible LCMS/MS method to quantify pentosidine and CML in human serum that should facilitate future studies examining circulating levels of these analytes in various disease states, including diabetes mellitus and renal insufficiency/ failure.

\section{SUPPLEMENTAL MATERIAL}

Supplemental material is available at The Journal of Applied Laboratory Medicine online.

Author Contributions: All authors confirmed they have contributed to the intellectual content of this paper and have met the following 4 requirements: (a) significant contributions to the conception and design, acquisition of data, or analysis and interpretation of data; (b) drafting or revising the article for intellectual content; (c) final approval of the published article; and (d) agreement to be accountable for all aspects of the article thus ensuring that questions related to the accuracy or integrity of any part of the article are appropriately investigated and resolved.

K.L. O'Grady, statistical analysis; E. Atkinson, statistical analysis; S.J. Achenbach, statistical analysis; A. Tweed, administrative support, provision of study material or patients; T. Volkman, provision of study material or patients.

Authors' Disclosures or Potential Conflicts of Interest: Upon manuscript submission, all authors completed the author disclosure form. Disclosures and/or potential conflicts of interest:

Employment or Leadership: None declared. Consultant or Advisory Role: None declared. Stock Ownership: None declared. Honoraria: None declared. Research Funding: NIH grants AR027065 and UL1TR002377. Expert Testimony: None declared. Patents: None declared.

Role of Sponsor: The funding organizations played no role in the design of study, choice of enrolled patients, review and interpretation of data, preparation of manuscript, or final approval of manuscript. 


\section{REFERENCES}

1. Chaudhuri J, Bains Y, Guha S, Kahn A, Hall D, Bose N, et al. The role of advanced glycation end products in aging and metabolic diseases: bridging association and causality. Cell Metab 2018;28:337-52.

2. Volpe CMO, Villar-Delfino PH, Dos Anjos PMF, NogueiraMachado JA. Cellular death, reactive oxygen species (ROS) and diabetic complications. Cell Death Dis 2018;9: 119.

3. Vlassara H. Recent progress in advanced glycation end products and diabetic complications. Diabetes 1997;46 Suppl 2:S19-25.

4. Vlassara H, Palace MR. Glycoxidation: the menace of diabetes and aging. Mt Sinai J Med 2003;70:232-41.

5. Stirban A, Gawlowski T, Roden M. Vascular effects of advanced glycation endproducts: clinical effects and molecular mechanisms. Mol Metab 2014;3:94-108.

6. Fishman SL, Sonmez H, Basman C, Singh V, Poretsky L. The role of advanced glycation end-products in the development of coronary artery disease in patients with and without diabetes mellitus: a review. Mol Med 2018; 24:59.

7. Taneda S, Monnier VM. Elisa of pentosidine, an advanced glycation end product, in biological specimens. Clin Chem 1994;40:1766-73.

8. Odetti P, Fogarty J, Sell DR, Monnier VM. Chromatographic quantitation of plasma and erythrocyte pentosidine in diabetic and uremic subjects. Diabetes 1992;41:153-9.

9. Nowotny K, Jung T, Hohn A, Weber D, Grune T. Advanced glycation end products and oxidative stress in type 2 diabetes mellitus. Biomolecules 2015;5:194-222.

10. Ashraf JM, Ahmad S, Choi I, Ahmad N, Farhan M, Tatyana $\mathrm{G}$, Shahab U. Recent advances in detection of ages: Immunochemical, bioanalytical and biochemical approaches. IUBMB Life 2015;67:897-913.

11. Levey AS, Stevens LA, Schmid CH, Zhang YL, Castro AF 3rd, Feldman $\mathrm{HI}$, et al. A new equation to estimate glomerular filtration rate. Ann Intern Med 2009;150: 604-12.

12. Meerwaldt R, Graaff R, Oomen PHN, Links TP, Jager JJ, Alderson NL, et al. Simple non-invasive assessment of advanced glycation endproduct accumulation. Diabetologia 2004;47:1324-30.

13. Wagner Z, Wittmann I, Mazak I, Schinzel R, Heidland A, Kientsch-Engel R, Nagy J. N(epsilon)-(carboxymethyl)lysine levels in patients with type 2 diabetes: Role of renal function. Am J Kidney Dis 2001;38:785-91.

14. Teerlink T, Barto R, Ten Brink HJ, Schalkwijk CG. Measurement of nepsilon-(carboxymethyl)lysine and nepsilon-(carboxyethyl)lysine in human plasma protein by stable-isotope-dilution tandem mass spectrometry. Clin Chem 2004;50:1222-8.

15. Ni J, Yuan X, Gu J, Yue X, Gu X, Nagaraj RH, Crabb JW. Plasma protein pentosidine and carboxymethyllysine, biomarkers for age-related macular degeneration. Mol Cell Proteomics 2009;8:1921-33.

16. Rankin NJ, Burgess K, Weidt S, Wannamethee G, Sattar N, Welsh P. High-throughput quantification of carboxymethyl lysine in serum and plasma using highresolution accurate mass orbitrap mass spectrometry. Ann Clin Biochem 2019;56:397-407.

17. Thornalley PJ, Rabbani N. Detection of oxidized and glycated proteins in clinical samples using mass spectrometry-a user's perspective. Biochim Biophys Acta 2014;1840:818-29.

18. Rabbani N, Ashour A, Thornalley PJ. Mass spectrometric determination of early and advanced glycation in biology. Glycoconj J 2016;33:553-68.

19. Scheijen JL, van de Waarenburg MP, Stehouwer $C D$, Schalkwijk CG. Measurement of pentosidine in human plasma protein by a single-column high-performance liquid chromatography method with fluorescence detection. J Chromatogr B Analyt Technol Biomed Life Sci 2009;877:610-4.

20. Haddad M, Knani I, Bouzidi H, Berriche O, Hammami M, Kerkeni M. Plasma levels of pentosidine, carboxymethyllysine, soluble receptor for advanced glycation end products, and metabolic syndrome: the metformin effect. Dis Markers 2016;2016:1-8.

21. Ghanem AA, Elewa A, Arafa LF. Pentosidine and ncarboxymethyl-lysine: biomarkers for type 2 diabetic retinopathy. Eur J Ophthalmol 2011;21:48-54.

22. Kerkeni M, Weiss IS, Jaisson S, Dandana A, Addad F, Gillery P, Hammami M. Increased serum concentrations of pentosidine are related to presence and severity of coronary artery disease. Thromb Res 2014;134:633-8.

23. Yoshida N, Okumura K, Aso Y. High serum pentosidine concentrations are associated with increased arterial stiffness and thickness in patients with type 2 diabetes. Metabolism 2005;54:345-50.

24. Sugiyama S, Miyata T, Ueda Y, Tanaka H, Maeda K, Kawashima S, et al. Plasma levels of pentosidine in diabetic patients: An advanced glycation end product. J Am Soc Nephrol 1998;9:1681-8.

25. Weiss MF, Erhard P, Kader-Attia FA, Wu YC, Deoreo PB, Araki $A$, et al. Mechanisms for the formation of glycoxidation products in end-stage renal disease. Kidney Int 2000:57:2571-85.

26. Hricik DE, Schulak JA, Sell DR, Fogarty JF, Monnier VM. Effects of kidney or kidney-pancreas transplantation on plasma pentosidine. Kidney Int 1993;43:398-403.

27. Tan AL, Forbes JM, Cooper ME. AGE, RAGE, and ROS in diabetic nephropathy. Semin Nephrol 2007;27:130-43.

28. Meerwaldt R, Hartog JW, Graaff R, Huisman RJ, Links TP, den Hollander NC, et al. Skin autofluorescence, a measure of cumulative metabolic stress and advanced glycation end products, predicts mortality in hemodialysis patients. JASN 2005;16:3687-93. 